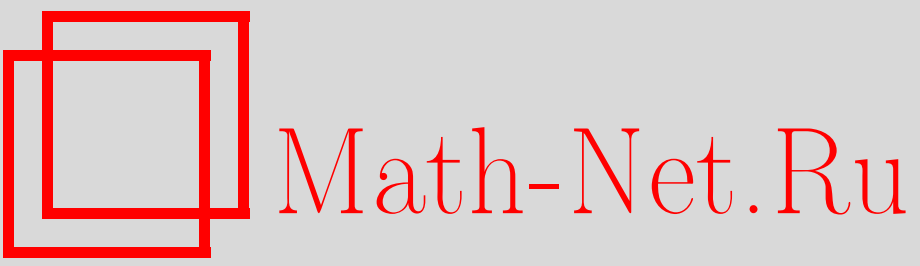

В. И. Алхимов, Эволюция в случайном гауссовом поле, ТМФ, 2004, том 139, номер 3, 512-528

DOI: https://doi.org/10.4213/tmf63

Использование Общероссийского математического портала Math-Net.Ru подразумевает, что вы прочитали и согласны с пользовательским соглашением

http://www.mathnet.ru/rus/agreement

Параметры загрузки:

IP: 54.198 .67 .100

26 апреля 2023 г., 12:51:08 
ТЕОРЕТИЧЕСКАЯ

И МАТЕМАТИЧЕСКАЯ

ФИЗИКА

Том 139, № 3

июнь, 2004

(C) 2004 r.

В.И. Алхимов*

\section{ЭВОЛЮЦИЯ В СЛУЧАЙНОМ ГАУССОВОМ ПОЛЕ}

Рассмотрен эволюционный процесс в случайном гауссовом поле $V(q)$ с нулевым средним, $\langle V(q)\rangle=0$, и корреляционной функцией $W\left(\left|\mathbf{q}-\mathbf{q}^{\prime}\right|\right) \equiv\left\langle V(q) V\left(q^{\prime}\right)\right\rangle$, где $\mathbf{q} \in \mathbb{R}^{d}, d$-размерность евклидова пространства $\mathbb{R}^{d}$. Для усредненной по всем реализациям случайного поля функции Грина $\left\langle G\left(\mathbf{q}, t ; \mathbf{q}_{0}\right)\right\rangle, t>0$, эволюционного уравнения с помощью формулы Фейнмана-Каца установлено интегральное уравнение, обладающее свойством инвариантности относительно непрерывной группы ренормировочных преобразований. Это свойство позволяет использовать ренормгрупповой метод для отыскания асимптотики функции $\left\langle G\left(\mathbf{q}, t ; \mathbf{q}_{0}\right)\right\rangle$, когда $\left|\mathbf{q}-\mathbf{q}_{0}\right| \rightarrow \infty$ и $t \rightarrow \infty$.

Ключевые слова: случайное поле, корреляционная функция, функция Грина, формула Фейнмана-Каца, ренормализационная группа.

\section{1. ПОСТАНОВКА ЗАДАЧИ}

Основная задача теории случайных (неупорядоченных) сред состоит в исследовании двух основных механизмов их эволюции: диссипации (например, диффузии или теплопроводности) и самоорганизации (обусловленной, например, воздействием внешних потенциальных полей или потоков) [1]-[4]. В данной работе используется модель ветвяшегося процесса с диффузией для изучения эволюции физической системы в стационарной случайной среде. Предполагается, что среда пространственно-однородна в среднем и в ней отсутствуют статистические корреляции между случайными параметрами, характеризуюшими неупорядоченность в достаточно удаленных друг от друга точках. Влияние среды на эволюционный процесс мы будем описывать посредством случайного потенциального поля $V(q), \mathbf{q} \in \mathbb{R}^{d}$, которое имеет гауссово распределение с нулевым средним, $\langle V(q)\rangle=0$, и корреляционной функцией

$$
W\left(\left|\mathbf{q}-\mathbf{q}^{\prime}\right|\right) \equiv\left\langle V(q) V\left(q^{\prime}\right)\right\rangle,
$$

где усреднение выполняется по всем реализациям случайного потенциала $V(q)$. В этом случае все характеристики потенциала $V(q)$ выражаются в терминах корреляционной функции $W(q)$, ограниченной на пространстве $\mathbb{R}^{d}$,

$$
|W(q)|<K, \quad K>0, \quad \forall \mathbf{q} \in \mathbb{R}^{d} .
$$

* Московский государственный областной университет, Москва, Россия. E-mail: vialkhim@mtu-net.ru 
Эта функция предполагается интегрируемой вместе со своей производной на $\mathbb{R}^{d}$ и достаточно быстро стремяшейся к нулю, когда $q / l \rightarrow \infty$, где $l$ - характеристическая длина, например, эффективный радиус корреляции функции $W(q)$, определяемый равенствами

$$
w l^{d}=\int_{\mathbb{R}^{d}} W(q) d \mathbf{q}, \quad w l^{d+1}=\int_{\mathbb{R}^{d}} q W(q) d \mathbf{q} .
$$

Эволюция во времени многих физических систем описывается уравнением

$$
\frac{\partial \rho}{\partial t}+H \rho=0
$$

где $\rho$ обозначает некоторую "плотность" системы, а $H$ - линейный оператор в пространстве непрерывных функций $\rho=\rho(\mathbf{q}, t), \mathbf{q} \in \mathbb{R}^{d}, t>0$. В случае, когда оператор $H$ имеет вид

$$
H=-D \sum_{j=1}^{d} \frac{\partial^{2}}{\partial q_{j}^{2}}+V(q), \quad D>0,
$$

уравнение (3) описьвает суперпозицию процессов диффузии (или распространения тепла) и ветвления (размножения и гибели) в стационарной среде. Если в уравнениях (3) и (4) формально положить $t=\hbar /\left(k_{\mathrm{B}} T\right), D=\hbar /(2 m), \hbar$ - постоянная Планка, $k_{\mathrm{B}}-$ постоянная Больцмана, $T$ - абсолютная температура, $m$ - масса частицы, то уравнение (3) будет описывать зависимость матрицы плотности для квантово-механической системы, находящейся в тепловом равновесии, от температуры [5]. Ради общности изложения мы не будем конкретизировать изучаемую модель физической системы. Поскольку в рассматриваемой модели взаимодействуют два механизма эволюции, то, очевидно, возможны два предельных режима, в каждом из которых преобладает один из указанных процессов. Эту ситуацию можно описать с помощью безразмерного параметра

$$
a=\frac{w l^{4}}{D^{2}}
$$

следуюшим образом. Если $a \ll 1$, то в общем процессе эволюции системы преобладает механизм диффузии. В случае $a \gg 1$ превалирует эффект ветвления. Так как последний случай подробно исследован в работе [6], то ниже мы рассмотрим процесс лишь при $a \ll 1$.

Объектом нашего исследования будет являться функция

$$
g(r, t)=\left\langle G\left(\mathbf{q}_{0}+\mathbf{r}, t ; \mathbf{q}_{0}\right)\right\rangle \quad \forall\left\{\mathbf{q}_{0}, \mathbf{r}\right\} \in \mathbb{R}^{d},
$$

где $G\left(\mathbf{q}, t ; \mathbf{q}_{0}\right)$ обозначает функцию Грина уравнения (3) или ядро оператора $e^{-t H}$ с оператором $H$, определенньм в (4). С помошью формулы Фейнмана-Каца [7]-[9] функцию Грина можно записать в виде

$$
G\left(\mathbf{q}, t ; \mathbf{q}_{0}\right)=\lim _{N \rightarrow \infty}(4 \pi D \tau)^{-N d / 2} \int_{\mathbb{R}^{d}} \cdots \int_{\mathbb{R}^{d}} e^{-S_{N}} \prod_{m=1}^{N-1} d \mathbf{q}_{m}
$$


где $\tau=t / N, t_{n}=n \tau, n=1,2, \ldots, N, \mathbf{q}_{n}=\mathbf{q}\left(t_{n}\right), \mathbf{q}_{0}=\mathbf{q}(0), \mathbf{q}=\mathbf{q}(t)$,

$$
S_{N}=\sum_{n=1}^{N}\left[\frac{\left(\mathbf{q}_{n}-\mathbf{q}_{n-1}\right)^{2}}{4 D \tau}+\tau V\left(\mathbf{q}_{n}\right)\right] .
$$

Далее мы воспользуемся формулой (7), теоремой Фубини и равенством

$$
\left\langle\exp \left[-\tau \sum_{n=1}^{N} V\left(q_{n}\right)\right]\right\rangle=A_{N}(t) \exp \left[\tau^{2} \sum_{0 \leqslant m} \sum_{<n \leqslant N} W\left(\left|\mathbf{q}_{n}-\mathbf{q}_{m}\right|\right)\right],
$$

в котором

$$
A_{N}(t)=\exp \left[\frac{t^{2}}{N}\left(\frac{1}{2} W(0)-\frac{1}{N} \sum_{n=1}^{N} W\left(\left|\mathbf{q}_{n}-\mathbf{q}_{0}\right|\right)\right)\right] .
$$

Вследствие ограниченности функции $W(q)$ имеем

$$
\lim _{N \rightarrow \infty} A_{N}(t)=1 .
$$

Тогда, как нетрудно видеть, искомую функцию $g(r, t)$ можно привести к виду

$$
g(r, t)=\lim _{N \rightarrow \infty} \frac{1}{(2 \pi)^{d}} \int e^{i \mathbf{r p}} g_{N}(p) d \mathbf{p}
$$

где

$$
\begin{aligned}
g_{N}(p) & =\int P_{1 N} \prod_{n=1}^{N} \theta_{p}\left(r_{n}\right) d \mathbf{r}_{n}, \\
P_{m n} & =\prod_{m \leqslant i \leqslant j \leqslant n} \prod_{j \leqslant n}\left(1+\varepsilon_{i j}\right), \\
\varepsilon_{i j}=\varepsilon\left(\left|\mathbf{q}_{j}-\mathbf{q}_{i-1}\right|\right) & =\varepsilon\left(\left|\sum_{i \leqslant k \leqslant j} \mathbf{r}_{k}\right|\right), \quad \mathbf{r}_{k}=\mathbf{q}_{k}-\mathbf{q}_{k-1}, \\
\varepsilon(r) & =\exp \left[\tau^{2} W(r)\right]-1,
\end{aligned}
$$

а функция $\theta_{p}(r)$ имеет вид

$$
\theta_{p}(r)=(4 \pi D \tau)^{-d / 2} \exp \left[-i \mathbf{p r}-\frac{\mathbf{r}^{2}}{4 D \tau}\right] .
$$

Из условия (1), равенств (9)-(12) и формулы

$$
\Theta(p) \equiv \int \theta_{p}(r) d \mathbf{r}=\exp \left[-\tau D p^{2}\right]
$$

нетрудно получить следующую оценку:

$$
\left|g_{N}(p)\right|<\exp \left(\frac{1}{2} K t^{2}-D p^{2} t\right) .
$$

Следует отметить, что в соответствуюших соотношениях выполняются два независимых функциональных интегрирования: одно - по пространству траекторий $\left\{\mathbf{q}: \mathbb{R}^{+} \rightarrow\right.$ $\left.\mathbb{R}^{d}\right\}$, другое - по всем реализациям случайного потенциала $V(q)[10]$. 


\section{2. ОСНОВНОЕ УРАВНЕНИЕ}

В этом разделе мы установим уравнение для величины $g(r, t)$, используя при этом некоторые результаты работ [11]-[13]. С этой целью в равенствах (8)-(10) воспользуемся разложением Майера произведения $P_{1 N}$ в ряд по $\varepsilon_{i j}$ :

$$
P_{1 N}=1+\sum_{i \leqslant j} \varepsilon_{i j}+\sum_{i \leqslant j} \sum_{k \leqslant l} \varepsilon_{i j} \varepsilon_{k l}+\sum_{i \leqslant j} \sum_{k \leqslant l} \sum_{m \leqslant n} \varepsilon_{i j} \varepsilon_{k l} \varepsilon_{m n}+\cdots,
$$

где индексы в каждом из сомножителей $\varepsilon_{i j}, \varepsilon_{k l}, \varepsilon_{m n}, \ldots$ располагаются в порядке неубывания, т.е. $i \leqslant j, k \leqslant l, m \leqslant n, \ldots$, и, кроме того, первые индексы этих сомножителей в каждом члене указанной суммы тоже образуют неубывающую последовательность, т.е. $i \leqslant k \leqslant m \leqslant \cdots$, причем $(i, j) \neq(k, l),(i, j) \neq(m, n),(k, l) \neq(m, n), \ldots$ Сомножители $\varepsilon_{i j}$ и $\varepsilon_{k l}$ назовем связанными, если $i \leqslant k \leqslant j$, и несвязанными, если $j<k$. Любое произведение сомножителей в (15), расположенных в порядке неубывания их первых индексов, будем называть комплексом, если в этом произведении каждый сомножитель, начиная со второго, связан по крайней мере с одним из предшествуюших сомножителей. Очевидно, любой член суммы в (15) является либо комплексом, либо распадается на произведение некоторого числа комплексов. Обозначим теперь через $f_{i j}$ сумму всех комплексов, зависяших от векторов $\mathbf{r}_{i}, \mathbf{r}_{i+1}, \ldots, \mathbf{r}_{j}, i \leqslant j$, и назовем ее блоком. Таким образом, по определению

$$
\begin{aligned}
& f_{i j}=\varepsilon_{i j} P_{i j}^{\prime}+\sum_{i<k \leqslant l<j} \varepsilon_{i l} \varepsilon_{k j} P_{i k-1} P_{k l} P_{l+1 j}+ \\
& +\sum_{i<k<l \leqslant m<n<j} \sum_{i n}\left(\varepsilon_{i n} \varepsilon_{k m} \varepsilon_{l j}+\varepsilon_{i m} \varepsilon_{k j} \varepsilon_{l n}+\varepsilon_{i m} \varepsilon_{k n} \varepsilon_{l j}\right) P_{i k-1} P_{k l-1} P_{l m} P_{m+1} P_{n+1 j}+ \\
& \quad+\varepsilon_{i l} \varepsilon_{k n} \varepsilon_{m j} P_{i k-1} P_{k l} P_{l+1 m-1} P_{m n} P_{n+1}+\cdots,
\end{aligned}
$$

где произведение $\varepsilon_{i j} P_{i j}^{\prime}$ определяется равенством

$$
\varepsilon_{i j} P_{i j}^{\prime}=\varepsilon_{i j} P_{i j}\left(1+\varepsilon_{i j}\right)^{-1} .
$$

В правой части (16) под знаком первой суммы фигурирует всего лишь один представитель комплексов второго порядка по $\varepsilon_{i j}$, в то время как под знаком второй суммы находятся четыре неэквивалентных представителя комплексов третьего порядка относительно $\varepsilon_{i j}$. Если обозначить через $N_{n}$ полное число неэквивалентных представителей комплексов $n$-го порядка по $\varepsilon_{i j}$, то оказывается, что $N_{4}=27$. В обшем случае для $N_{n}$ в работе [14] установлено рекуррентное соотношение, из которого следует, что $N_{n}=O((2 n-1) ! !)$ при $n \rightarrow \infty$. Тогда нетрудно видеть, что произведение $P_{1 N}$ можно разложить по блокам $f_{i j}$,

$$
\begin{gathered}
P_{1 N}=1+\sum_{1 \leqslant i \leqslant i+k \leqslant N} f_{i i+k}+\sum_{1 \leqslant i_{1} \leqslant i_{1}+k_{1}<i_{2} \leqslant i_{2}+k_{2} \leqslant N} \sum_{i_{1} i_{1}+k_{1}} f_{i_{2} i_{2}+k_{2}}+\cdots+ \\
+\sum_{1 \leqslant i_{1} \leqslant i_{1}+k_{1}<i_{2} \leqslant i_{2}+k_{2}<\cdots<i_{m} \leqslant i_{m}+k_{m} \leqslant N} \sum_{i_{1} i_{1}+k_{1}} f_{i_{2}} i_{2}+k_{2} \ldots f_{i_{m}} i_{m}+k_{m} \\
\cdots
\end{gathered}
$$


так что каждый член суммы в (18) является либо блоком, либо произведением некоторого числа блоков. Теперь воспользуемся разложением (18) в равенстве (9) и затем проинтегрируем каждый член полученного ряда. Находим, что

$$
\begin{aligned}
\int \prod_{n=1}^{N} \theta_{p}\left(r_{n}\right) d \mathbf{r}_{n} & =\Theta^{N}(p), \\
\int\left[\sum_{1 \leqslant i \leqslant i+k \leqslant N} f_{i i+k}\right] \prod_{n=1}^{N} \theta_{p}\left(r_{n}\right) d \mathbf{r}_{n} & =\sum_{n=0}^{N}(N-n+1) \Theta^{N-n}(p) f_{n}(p),
\end{aligned}
$$

где

$$
f_{0}(p) \equiv 0, \quad f_{n}(p)=\int f_{1 n} \prod_{m=1}^{n} \theta_{p}\left(r_{m}\right) d \mathbf{r}_{m} \quad \forall n \geqslant 1,
$$

$f_{1 n}-$ блок, составленный из всех комплексов, зависящих лишь от векторов $\mathbf{r}_{1}, \mathbf{r}_{2}, \ldots, \mathbf{r}_{n}$, а $(N-n+1)$ - число способов размешения одного отрезка, состояшего из $n$ звеньев, внутри отрезка, содержащего $N$ звеньев. Наконец,

$$
\begin{aligned}
& \int \sum_{1 \leqslant i_{1} \leqslant i_{1}+k_{1}<i_{2} \leqslant i_{2}+k_{2}<\ldots \ldots<i_{m} \leqslant i_{m}+k_{m} \leqslant N} f_{i_{1} i_{1}+k_{1}} f_{i_{2} i_{2}+k_{2}} \ldots f_{i_{m} i_{m}+k_{m}}= \\
& =\sum_{n=0}^{N} \frac{(N-n+m) !}{(N-n) ! m !} \Theta^{N-n}(p) F_{n}^{(m)}(p),
\end{aligned}
$$

где

$$
F_{n}^{(0)}(p) \equiv 0, \quad F_{n}^{(m)}(p)=\sum_{n_{1}+\cdots+n_{m}=n} \ldots \sum_{n_{1}} f_{n_{1}}(p) \ldots f_{n_{m}}(p), \quad \forall m \geqslant 1,
$$

а множитель $(N-n+m) ! /((N-n) ! m !)$ равен числу способов размешения $m$ частичных отрезков, состоящих последовательно из $n_{1}, n_{2}, \ldots, n_{m}$ звеньев , общее число которых равно $n$ и которые расположены внутри одного отрезка, состояшего из $N$ звеньев, таким образом, чтобы при этом сохранялась последовательность расположения частичных отрезков. В результате величину $g_{N}(p)$ можно представить в виде

$$
g_{N}(p)=\sum_{m \geqslant 0} \sum_{n=0}^{N} \frac{(N-n+m) !}{(N-n) ! m !} \Theta^{N-n}(p) F_{n}^{(m)}(p) .
$$

По формуле Коши

$$
\frac{1}{2 \pi i} \oint_{C} d z z^{n_{1}+n_{2}+\cdots+n_{m}-n-1}=\left\{\begin{array}{lll}
1, & \text { если } & \sum_{j=1}^{m} n_{j}=n, \\
0, & \text { если } & \sum_{j=1}^{m} n_{j} \neq n,
\end{array}\right.
$$

где замкнутый контур $C$ охватывает начало координат на комплексной $z$-плоскости, равенство в (20) приводится к виду

$$
F_{n}^{(m)}(p)=\frac{1}{2 \pi i} \oint_{C} \frac{d z}{z^{n+1}} f^{m}(p, z),
$$


где

$$
f(p, z) \equiv \sum_{n \geqslant 0} z^{n} f_{n}(p)
$$

Используя выражение (22), соотношение (21) запишем в виде

$$
g_{N}(p)=\frac{1}{2 \pi i} \oint_{C} \frac{d z}{z^{N+1}} \sum_{m \geqslant 0} \sum_{n=0}^{N} \frac{(N-n+m) !}{(N-n) ! m !}[z \Theta(p)]^{N-n} f^{m}(p, z)
$$

где суммирование по $m$ можно распространить формально от 0 до $\infty$, а по $n-$ от $-\infty$ до $N$, не оказывая влияния при этом на конечный результат. Принимая во внимание последнее замечание и формулу

$$
(1-x-y)^{-1}=\sum_{m=0}^{\infty} \sum_{n=0}^{\infty} \frac{(m+n) !}{m ! n !} x^{m} y^{n}, \quad|x+y|<1,
$$

приведем соотношение (24) к виду

$$
g_{N}(p)=\frac{1}{2 \pi i} \oint_{C} \frac{d z}{z^{N+1}}[1-z \Theta(p)-f(p, z)]^{-1}
$$

где замкнутый контур $C$, охватывающий начало координат $z=0$, выбран так, чтобы выполнялось неравенство

$$
|z \Theta(p)+f(p, z)|<1
$$

Если определить производящую функцию

$$
g(p, z) \equiv \sum_{N \geqslant 0} z^{N} g_{N}(p)
$$

то из равенства (25) следует уравнение

$$
g^{-1}(p, z)=g_{0}^{-1}(p, z)-f(p, z)
$$

в котором

$$
g_{0}^{-1}(p, z)=1-z \Theta(p)
$$

Отметим, что согласно условию (14) ряд в (27) абсолютно сходится по крайней мере в области $|z|<1$. Уравнение (28) имеет вид известного уравнения Дайсона и в решении рассматриваемой задачи играет главную роль. Кроме того, необходимо установить непосредственную связь между производящими функциями $f(p, z)$ и $g(p, z)$, чтобы это уравнение было замкнутым. Для этого подставим определенное в (16) выражение для $f_{1 n}$ в равенство $(19)$ и запишем величину $\varepsilon_{m n}$ в следуюшей форме:

$$
\varepsilon_{m n}=\frac{1}{(2 \pi)^{d}} \int \tilde{\varepsilon}(\mathbf{k}) \exp \left[i \mathbf{k} \sum_{j=m}^{n} \mathbf{r}_{j}\right] d \mathbf{k}
$$


где

$$
\tilde{\varepsilon}(\mathbf{k})=\int e^{-i \mathbf{k r}} \varepsilon(\mathbf{r}) d \mathbf{r}
$$

в соответствии с определениями $\varepsilon_{m n}$ и $\varepsilon(r)$ в $(11)$. Выполняя затем в $(19)$ интегрирование по $\mathbf{r}_{m}, 1 \leqslant m \leqslant n$, находим

$$
\begin{aligned}
f_{n}(p)= & \frac{1}{(2 \pi)^{d}} \int \tilde{\varepsilon}(\mathbf{k}) g_{n}^{\prime}(\mathbf{p}-\mathbf{k}) d \mathbf{k}+\sum_{1<i \leqslant j<n} \frac{1}{(2 \pi)^{2 d}} \times \\
& \times \int \tilde{\varepsilon}(\mathbf{k}) \tilde{\varepsilon}\left(\mathbf{k}^{\prime}\right) g_{i-1}(\mathbf{p}-\mathbf{k}) g_{j-i+1}\left(\mathbf{p}-\mathbf{k}-\mathbf{k}^{\prime}\right) g_{n-j}\left(\mathbf{p}-\mathbf{k}^{\prime}\right) d \mathbf{k} d \mathbf{k}^{\prime}+ \\
& +\sum_{1<i<j \leqslant k<l<n} \sum_{k} \frac{1}{(2 \pi)^{3 d}} \int \tilde{\varepsilon}(\mathbf{k}) \tilde{\varepsilon}\left(\mathbf{k}^{\prime}\right) \tilde{\varepsilon}\left(\mathbf{k}^{\prime \prime}\right)\left\{g_{i-1}(\mathbf{p}-\mathbf{k}) g_{j-i}\left(\mathbf{p}-\mathbf{k}-\mathbf{k}^{\prime}\right) \times\right. \\
& \times g_{k-j+1}\left(\mathbf{p}-\mathbf{k}-\mathbf{k}^{\prime}-\mathbf{k}^{\prime \prime}\right)\left[g_{l-k}\left(\mathbf{p}-\mathbf{k}-\mathbf{k}^{\prime \prime}\right) g_{n-l}\left(\mathbf{p}-\mathbf{k}^{\prime \prime}\right)+\right. \\
& \left.+g_{l-k}\left(\mathbf{p}-\mathbf{k}^{\prime}-\mathbf{k}^{\prime \prime}\right) g_{n-l}\left(\mathbf{p}-\mathbf{k}^{\prime}\right)+g_{l-k}\left(\mathbf{p}-\mathbf{k}^{\prime}-\mathbf{k}^{\prime \prime}\right) g_{n-l}\left(\mathbf{p}-\mathbf{k}^{\prime \prime}\right)\right]+ \\
& +g_{i-1}(\mathbf{p}-\mathbf{k}) g_{j-i}\left(\mathbf{p}-\mathbf{k}-\mathbf{k}^{\prime}\right) g_{k-j+1}\left(\mathbf{p}-\mathbf{k}^{\prime}\right) \times \\
& \left.\times g_{l-k}\left(\mathbf{p}-\mathbf{k}^{\prime}-\mathbf{k}^{\prime \prime}\right) g_{n-l}\left(\mathbf{p}-\mathbf{k}^{\prime \prime}\right)\right\} d \mathbf{k} d \mathbf{k}^{\prime} d \mathbf{k}^{\prime \prime}+\cdots
\end{aligned}
$$

где функция $g_{N}^{\prime}(p)$ определена выражением (9), в котором произведение $P_{1 N}$ должно быть заменено на $P_{1 N}^{\prime}$. Принимая во внимание равенства (17) и (11), а также учитьвая ограниченность функции $W(q)$, нетрудно видеть, что $\left|g_{n}^{\prime}(\mathbf{p})-g_{n}(\mathbf{p})\right|=O\left(\tau^{2}\right)$, когда $\tau \rightarrow 0$. Поскольку для решения задачи нам предстоит в дальнейшем выполнить предельный переход, при котором $\tau \rightarrow 0$, то отсюда следует, что функции $g_{n}^{\prime}(\mathbf{p})$ и $g_{n}(\mathbf{p})$ можно принять совпадаюшими всюду. Если теперь подставить выражение (32) в равенство (23), то после надлежашего суммирования получим для $f(p, z)$ бесконечный ряд

$$
\begin{aligned}
f(p, z)= & \frac{1}{(2 \pi)^{d}} \int \tilde{\varepsilon}(\mathbf{k}) g(\mathbf{p}-\mathbf{k}, z) d \mathbf{k}+ \\
& +\frac{1}{(2 \pi)^{2 d}} \int \tilde{\varepsilon}(\mathbf{k}) \tilde{\varepsilon}\left(\mathbf{k}^{\prime}\right) g(\mathbf{p}-\mathbf{k}, z) g\left(\mathbf{p}-\mathbf{k}-\mathbf{k}^{\prime}, z\right) g\left(\mathbf{p}-\mathbf{k}^{\prime}, z\right) d \mathbf{k} d \mathbf{k}^{\prime}+ \\
& +\frac{1}{(2 \pi)^{3 d}} \int \tilde{\varepsilon}(\mathbf{k}) \tilde{\varepsilon}\left(\mathbf{k}^{\prime}\right) \tilde{\varepsilon}\left(\mathbf{k}^{\prime \prime}\right)\left\{g(\mathbf{p}-\mathbf{k}, z) g\left(\mathbf{p}-\mathbf{k}-\mathbf{k}^{\prime}, z\right) \times\right. \\
& \times g\left(\mathbf{p}-\mathbf{k}-\mathbf{k}^{\prime}-\mathbf{k}^{\prime \prime}, z\right)\left[g\left(\mathbf{p}-\mathbf{k}-\mathbf{k}^{\prime \prime}, z\right) g\left(\mathbf{p}-\mathbf{k}^{\prime \prime}, z\right)+\right. \\
& \left.+g\left(\mathbf{p}-\mathbf{k}^{\prime}-\mathbf{k}^{\prime \prime}, z\right) g\left(\mathbf{p}-\mathbf{k}^{\prime}, z\right)+g\left(\mathbf{p}-\mathbf{k}^{\prime}-\mathbf{k}^{\prime \prime}, z\right) g\left(\mathbf{p}-\mathbf{k}^{\prime \prime}, z\right)\right]+ \\
& +g(\mathbf{p}-\mathbf{k}, z) g\left(\mathbf{p}-\mathbf{k}-\mathbf{k}^{\prime}, z\right) g\left(\mathbf{p}-\mathbf{k}^{\prime}, z\right) g\left(\mathbf{p}-\mathbf{k}^{\prime}-\mathbf{k}^{\prime \prime}, z\right) \times \\
& \left.\times g\left(\mathbf{p}-\mathbf{k}^{\prime \prime}, z\right)\right\} d \mathbf{k} d \mathbf{k}^{\prime} d \mathbf{k}^{\prime \prime}+\cdots,
\end{aligned}
$$

посредством которого описывается искомая связь между функциями $f(p, z)$ и $g(p, z)$.

Таким образом, соотношения (28) и (33) образуют замкнутую систему уравнений относительно искомой функции $g(p, z)$. Ряд в $(33)$ удобно представить в символической форме

$$
f(p, z)=\sum_{n \geqslant 1} N_{n} \int\{\tilde{\varepsilon}(\mathbf{k})\}^{n}\{g(|\mathbf{p}-\mathbf{k}|, z)\}^{2 n-1}\left\{\frac{d \mathbf{k}}{(2 \pi)^{d}}\right\}^{n}
$$


для того чтобы продемонстрировать инвариантность уравнения (28) относительно мультипликативных преобразований

$$
g \rightarrow g^{\prime}=\alpha g, \quad g_{0} \rightarrow g_{0}^{\prime}=\alpha g_{0}, \quad \tilde{\varepsilon} \rightarrow \tilde{\varepsilon}^{\prime}=\alpha^{-2} \tilde{\varepsilon}, \quad f \rightarrow f^{\prime}=\alpha^{-1} f,
$$

где $\alpha$ - отличный от нуля параметр. Эти преобразования образуют непрерывную группу, называемую обычно ренормгруппой (РГ), что указывает на возможность применения ренормгруппового метода к исследуемой здесь проблеме.

Обратимся теперь к равенствам (11) и (31), согласно которым

$$
\tilde{\varepsilon}(k) \sim \tau^{2} u(k), \quad \tau \rightarrow 0,
$$

где

$$
u(k)=\int_{\mathbb{R}^{d}} e^{-i \mathbf{k r}} W(r) d \mathbf{r}
$$

- пространственный спектр случайного потенциала $V(k)$, удовлетворяющий условию

$$
0<u(k) \leqslant u(0)=w l^{d}
$$

согласно (2). Ранее высказанное предположение о поведении функции $W(r)$ при больших значениях $r$ равносильно предположению о регулярности пространственного спектра $u(k)$ в точке $k=0$. Кроме того, вследствие предполагаемой интегрируемости производной $d W(r) / d r$, из леммы Римана-Лебега вытекает, что

$$
u(k)<\frac{C}{k^{2}},
$$

когда $k \rightarrow \infty, C>0$. В дальнейшем через $C$ мы будем обозначать положительную постоянную, которая в разных формулах может принимать различные значения. Далее, из соотношений (13), (33) и (36) следует, что в случае $\tau \rightarrow 0$ и $\operatorname{Im} p=0$ условие (26) вырож дается в неравенство $|z|<1$. В связи с этим введем новую переменную $E(\operatorname{Re} E>0)$ посредством равенства

$$
z=z(\tau) \equiv e^{-E \tau}
$$

и запишем уравнение (28) в виде

$$
\begin{aligned}
{[\tau g(p, z(\tau))]^{-1}=} & \tau^{-1}\left\{1-\exp \left[-\tau\left(E+D p^{2}\right)\right]\right\}- \\
& -\sum_{n \geqslant 1} N_{n} \int\left\{\tau^{-2} \tilde{\varepsilon}(\mathbf{k})\right\}^{n}\{\tau g(|\mathbf{p}-\mathbf{k}|, z(\tau))\}^{2 n-1}\left\{\frac{d \mathbf{k}}{(2 \pi)^{d}}\right\}^{n}
\end{aligned}
$$

принимая во внимание РГ-свойство (35). Вследствие неравенства

$$
|\tau g(p, z(\tau))|<\tau\left[1-e^{-\tau E}\right]^{-1} \exp \left(\frac{1}{2} K t^{2}-D p^{2} t\right),
$$


вытекаюшего из формул (14) и (27), аналитическая в области $|z|<1$ функция $(1-z) \times$ $g(p, z)$ является ограниченной в этой области, и поэтому существует предел

$$
\lim _{\tau \rightarrow 0} \tau g(p, z(\tau)) \equiv \varphi(p, E) .
$$

Вьполняя предельный переход в уравнении (40) при $\tau \rightarrow 0$ и учитывая при этом соотношения (36) и (42), получим для функции $\varphi(p, E)$ следуюшее уравнение:

$$
\varphi^{-1}(p, E)=E+D p^{2}-F(p, E ; u ; \varphi),
$$

где величина $F(p, E ; u ; \varphi)$ согласно (34) выглядит символически как

$$
F(p, E ; u ; \varphi)=\sum_{n \geqslant 1} N_{n} \int\{\varphi(|\mathbf{p}-\mathbf{k}|, E)\}^{2 n-1}\left\{\frac{u(k) d \mathbf{k}}{(2 \pi)^{d}}\right\}^{n} .
$$

Теперь мы воспользуемся формулой обрашения для равенства (27), чтобы выразить функцию $g_{N}(p)$ через $g(p, z)$, и затем перейдем к пределу в (8), учитывая при этом равенства (39) и (42). В результате получим

$$
\lim _{N \rightarrow \infty} g_{N}(p)=\frac{1}{2 \pi i} \int_{C-i \infty}^{C+i \infty} e^{E t} \varphi(p, E) d E
$$

где контур интегрирования расположен справа от сингулярных точек функции $\varphi(p, E)$. Учитывая равенство (45) в формуле (8), получим

$$
g(r, t)=\frac{1}{2 \pi i} \int_{C-i \infty}^{C+i \infty} e^{E t} \psi(r, E) d E,
$$

где

$$
\psi(r, E)=\frac{1}{(2 \pi)^{d}} \int e^{i \mathbf{r} \mathbf{p}} \varphi(p, E) d \mathbf{p} .
$$

Таким образом, определение функции $g(r, t)$ сводится к решению уравнения (43). Эта проблема очень трудна, поэтому мы займемся здесь более простой задачей, а именно определением асимптотического поведения функции $g(r, t)$ при $r \rightarrow \infty$ и $t \rightarrow \infty$. Из формул (46) и (47) следует, что в этом случае необходимо знать поведение функции $\varphi(p, E)$ при малых значениях $p$ и $\left|E-E_{0}\right|$, где $E_{0}$ - наиболее удаленная вправо вещественная особая точка функции $\varphi(0, E)$, определяемая согласно (43) уравнением

$$
E_{0}-F\left(0, E_{0} ; u ; \varphi\right) \equiv 0 .
$$

Далее, обозначим через $p_{1,2} \equiv \pm i \xi, \operatorname{Re} \xi>0$, ближайшие к началу $p=0$ корни уравнения $\varphi^{-1}(p, E)=0$. Тогда, полагая $p=p_{1}$ в (43), получим тождество

$$
E+D p_{1}^{2}-F\left(p_{1}, E ; u ; \varphi\right) \equiv 0 .
$$

Из (48) и (49) вытекает следуюшая связь между $E-E_{0}$ и $p_{1}^{2}$ :

$$
E-E_{0}+D p_{1}^{2}+F\left(0, E_{0} ; u ; \varphi\right)-F\left(p_{1}, E ; u, \varphi\right) \equiv 0 .
$$


Наконец, с помощью тождества (49) преобразуем уравнение (43) к виду

$$
\varphi^{-1}=\varphi_{0}^{-1}+\Phi\left(\chi^{2}, \xi^{2} ; u ; \varphi\right)
$$

где

$$
\begin{gathered}
\varphi_{0}^{-1}=D \chi^{2}, \quad \chi^{2}=p^{2}+\xi^{2} \\
\Phi\left(\chi^{2}, \xi^{2} ; u ; \varphi\right)=F(i \xi, E ; u ; \varphi)-F(p, E ; u ; \varphi) .
\end{gathered}
$$

Система уравнений (50) и (51) составляет основу для отыскания асимптотики функции $g(r, t)$ при $r \rightarrow \infty$ и $t \rightarrow \infty$, если задана корреляционная функция $W(r)$.

\section{3. РГ-УРАВНЕНИЯ}

Представим искомую функцию $\varphi$ как

$$
\varphi=\varphi_{0} X
$$

где новая неизвестная величина $X$ удовлетворяет уравнению

$$
X^{-1}=1+\varphi_{0} \Phi\left(\chi^{2}, \xi^{2} ; u ; \varphi_{0} X\right)
$$

согласно (51) и (53). Предположим теперь, что при некотором не равном нулю значении $\chi^{2}=\lambda$ справедливо равенство

$$
\Phi\left(\lambda, \xi^{2} ; u ; \varphi_{0} X\right)=0,
$$

где $\lambda=\lambda\left(\xi^{2} ; u\right)$. Тогда безразмерную величину $X$ можно представить как функцию безразмерных переменных,

$$
X=X(x, y, b),
$$

где

$$
x=\frac{\chi^{2}}{\lambda}, \quad y=\frac{\xi^{2}}{\lambda}, \quad b=\frac{a}{\left(\lambda l^{2}\right)^{1-s}}, \quad s=\frac{d-2}{2},
$$

причем в точке $x=1$ выполняется условие нормировки

$$
X(1, y, b)=1 .
$$

Далее, умножим обе части уравнения (55) на $\alpha^{-1}$ и, учитывая РГ-свойство этого уравнения, запишем его в эквивалентной форме

$$
\left(X^{\prime}\right)^{-1}=1+\varphi_{0} \Phi\left(\chi^{2}, \xi^{2} ; u^{\prime} ; \varphi_{0} X^{\prime}\right)-\left(1-\alpha^{-1}\right),
$$

где $X^{\prime}=\alpha X, u^{\prime}=\alpha^{-2} u$. Теперь выберем значение $\chi^{2}=\lambda^{\prime}, \lambda^{\prime} \neq 0$, так, чтобы

$$
\Phi\left(\lambda^{\prime}, \xi^{2} ; u^{\prime} ; \varphi_{0} X^{\prime}\right)=D \lambda^{\prime}\left(1-\alpha^{-1}\right),
$$


т.е.

$$
\left[X^{\prime}\right]_{\chi^{2}=\lambda^{\prime}}=1 .
$$

Это означает, что РГ-свойство уравнения (55) можно записать в форме

$$
\alpha X(x, y, b)=X\left(\frac{x}{\nu}, \frac{y}{\nu} ; b \alpha^{-2} \nu^{s-1}\right),
$$

где $\nu=\lambda^{\prime} / \lambda$. Если в (59) положить $x=\nu$ и учесть условие нормировки (58), то

$$
\alpha^{-1}=X(\nu, y, b)
$$

а равенство (59) преобразуется к виду

$$
X(x, y, b)=X(\nu, y, b) X\left(\frac{x}{\nu}, \frac{y}{\nu}, b \nu^{s-1} X^{2}(\nu, y, b)\right) .
$$

Далее, возведем в квадрат обе части уравнения (60) и затем умножим его на $b x^{s-1}$. В результате находим, что величина

$$
B(x, y, b)=b x^{s-1} X^{2}(x, y, b)
$$

удовлетворяет уравнению

$$
B(x, y, b)=B\left(\frac{x}{\nu}, \frac{y}{\nu}, B(\nu, y, b)\right)
$$

с нормировочным условием

$$
B(1, y, b)=b .
$$

Уравнение (62) замкнуто и может быть решено в обшем виде. Однако для практических целей более удобно иметь дело с дифференшиальным уравнением Ли, соответствуюшим непрерывной РГ [15].

Чтобы получить уравнение Ли для функции $B(x, y, b)$, продифференцируем обе части уравнения (62) по $x$ и затем положим $\nu=x$. В результате получим

$$
x \frac{\partial B(x, y, b)}{\partial x}=\beta\left(\frac{y}{x}, B(x, y, b)\right),
$$

где

$$
\beta(y, b)=\left.\frac{\partial B(x, y, b)}{\partial x}\right|_{x=1},
$$

а равенство (63) играет роль граничного условия для уравнения (64). В частном случае, когда $y \rightarrow 0$, для функции

$$
B(x, b)=\lim _{y \rightarrow 0} B(x, y, b)
$$

уравнение (64) приводится к виду

$$
x \frac{\partial B(x, b)}{\partial x}=\beta(B(x, b)),
$$


где

$$
\beta(b)=\left.\frac{\partial B(x, b)}{\partial x}\right|_{x=1},
$$

а соответствующее граничное условие принимает форму

$$
B(1, b)=b
$$

Уравнение (67) совместно с (69) можно записать также в виде уравнения Гелл-МаннаЛоу

$$
\int_{b}^{B(x, b)} \beta^{-1}(\nu) d \nu=\ln x
$$

которое оказывается очень удобным для исследования поведения инвариантной величины $B(x, b)$ при малых значениях $x$, или $p^{2} \ll \lambda$.

Таким образом, согласно РГ-уравнениям $B(x, b)$ является эффективным параметром, характеризующим корреляцию в малой окрестности точек $p=0$ и $\xi=0$. Отсюда следует, что для определения асимптотического поведения функции

$$
X(x, b)=\lim _{y \rightarrow 0} X(x, y, b),
$$

когда $x \rightarrow 0$, необходимо знать свойства функции $\beta(b)$, определяемой уравнением (68). Обычно для вычисления функции $\beta(b)$ используется теория возмушений, в связи с чем реальную информацию о ее поведении удается получить лишь в малой окрестности точки $b=0$, где $\beta(0)=0$. Действительно, если $\beta(b)$ положительна в этой окрестности, то инвариантная величина $B(x, b)$ стремится к нулю, когда $x \rightarrow 0$, т.е. точка $b=0$ является стабильным нулем. Если же функция $\beta(b)$ отрицательна вблизи нуля, то $B(x, b)$ возрастает, когда $x \rightarrow 0$, что находится за пределами применимости теории возмушений. Согласно выражениям (61) и (71) $B(x, b) \rightarrow 0$ при $x \rightarrow 0$, если

$$
X(x, b)=o\left(x^{(1-s) / 2}\right), \quad x \rightarrow 0 .
$$

Отсюда следует, что в том случае, когда $X(x, b)=O\left(x^{\mu}\right)$ при $x \rightarrow 0$, условие

$$
1-s<2 \mu
$$

является необходимым для определения функции $\beta(b)$ в окрестности точки $b=0$. Однако величина $\mu$ не зависит от $y$, и поэтому функция $X(x, y, b)$ тоже будет вести себя как $O\left(x^{\mu}\right)$ при $x \rightarrow 0$. Но тогда в соответствии с равенством (54) искомая функция $\varphi$ должна вести себя в этом случае как $O\left(x^{\mu-1}\right)$.

Учитывая последнее замечание, обратимся к уравнению (55) и в его правой части рассмотрим поведение функции $\chi^{-2} \Phi\left(\chi^{2}, \xi^{2} ; u ; \varphi\right)$ для малых значений параметра $a$ и 
значений $p$, принадлежаших малой окрестности точки $p=p_{1}$. Для этого воспользуемся разложением функции $\Phi$, определяемым равенствами (53) и (44),

$$
\begin{aligned}
\Phi\left(\chi^{2}, \xi^{2} ; u ; \varphi\right) & =\frac{1}{(2 \pi)^{d}} \int\left[\varphi\left(\left|\mathbf{p}_{1}-\mathbf{k}\right|, E\right)-\varphi(|\mathbf{p}-\mathbf{k}|, E)\right] u(k) d \mathbf{k}+ \\
& +\frac{1}{(2 \pi)^{2 d}} \int\left[\varphi\left(\left|\mathbf{p}_{1}-\mathbf{k}\right|, E\right) \varphi\left(\left|\mathbf{p}_{1}-\mathbf{k}-\mathbf{k}^{\prime}\right|, E\right) \varphi\left(\left|\mathbf{p}_{1}-\mathbf{k}^{\prime}\right|, E\right)-\right. \\
& \left.-\varphi(|\mathbf{p}-\mathbf{k}|, E) \varphi\left(\left|\mathbf{p}-\mathbf{k}-\mathbf{k}^{\prime}\right|, E\right) \varphi\left(\left|\mathbf{p}-\mathbf{k}^{\prime}\right|, E\right)\right] u(k) u\left(k^{\prime}\right) d \mathbf{k} d \mathbf{k}^{\prime}+\cdots
\end{aligned}
$$

где указаны явно лишь члены первого и второго порядка относительно $u(k)$. Асимптотическая оценка подынтегральных функций в каждом члене ряда $(74)$, когда $\varphi(k, E)=$ $O\left(\left|k^{2}+\xi^{2}\right|^{\mu-1}\right)$ и $u(k)<C k^{-2}$ при $k \rightarrow \infty$, показывает, что сходимость интегралов в (74) будет обеспечена, если выполнено условие

$$
\mu<1-s
$$

Тогда условия (73) и (75) можно объединить в одно условие

$$
\mu<1-s<2 \mu,
$$

где $0 \leqslant s<1$ (или $2 \leqslant d<4$ ). Таким образом, для определения асимптотической формы функции $\varphi(p, E)$, когда $p \rightarrow 0$ и $E \rightarrow E_{0}$ (или $\xi \rightarrow 0$ ), необходимо найти значение $\mu=\mu(s)$, удовлетворяющее условию (76).

\section{4. АСИМПТОТИЧЕСКОЕ ПРИБЛИЖЕНИЕ}

В соответствии с вышесказанным примем в качестве исходного приближения для $\varphi(p, E)$ выражение

$$
\tilde{\varphi}(p, \xi)=A D^{-1}\left(\frac{L}{\chi}\right)^{1-\mu} K_{1-\mu}(L \chi), \quad l \ll L,
$$

в котором значения параметров $A$ и $\mu=\mu(s), 0 \leqslant s<1$, должны быть найдены, а использование функции Макдональда $K_{\nu}(z)$ обусловлено ее известными [16] асимптотическими свойствами

$$
K_{\nu}(z) \sim\left\{\begin{array}{ll}
2^{\nu-1} \Gamma(\nu) z^{-\nu}, & z \rightarrow 0, \\
\sqrt{\pi / 2 z} e^{-z}, & z \rightarrow \infty,
\end{array} \quad K_{-\nu}(z)=K_{\nu}(z), \quad \nu>0\right.
$$

адекватными свойствам решения уравнения (51). Здесь следует подчеркнуть, что выбор конкретной формы исходного приближения для функции $\varphi(p, E)$ неоднозначен, если нас интересует ее асимптотика при $\xi \rightarrow 0$ и $p \rightarrow 0$. Этот выбор определяется двумя требованиями. Во-первых, необходимо учесть вклад в величину $\tilde{\varphi}(p, \xi)$ только ближайшей к началу $p=0$ особой точки $p_{1}=i \xi$. Во-вторых, в соответствии с вышеприведенным анализом поведение функции $\tilde{\varphi}(p, \xi)$ в окрестности точки $p=p_{1}$ должно иметь вид

$$
\tilde{\varphi}(p, \xi)=O\left(\chi^{2(\mu-1)}\right), \quad \chi \rightarrow 0 .
$$


Но тогда, согласно (47) и (77), соответствуюшее приближение для функции $\psi(r, E)$ есть

$$
\tilde{\psi}(r, \xi)=(2 \pi)^{-d / 2} A D^{-1} R^{-2 \nu} \bar{K}_{\nu}(\xi R),
$$

где

$$
R^{2}=r^{2}+L^{2}, \quad \bar{K}_{\nu}(z)=z^{\nu} K_{\nu}(z), \quad \nu=\mu+s .
$$

Из свойства (79) и условия нормировки функции

$$
\widetilde{X}\left(\frac{\chi^{2}}{\lambda}, \frac{\xi^{2}}{\lambda}, b\right)=D \chi^{2} \tilde{\varphi}(p, \xi)
$$

в точке $\chi^{2}=\lambda$ следует, что

$$
A^{-1}=\lambda^{\mu} \bar{K}_{1-\mu}(L \sqrt{\lambda}), \quad L \sqrt{\lambda} \ll 1,
$$

откуда, в свою очередь, вытекает неравенство $l \sqrt{\lambda} \ll 1$. В рассматриваемом приближении согласно соотношениям (44) и (53) запишем функцию $\Phi\left(\chi^{2}, \xi^{2} ; u ; \tilde{\varphi}\right)$ в виде

$$
\begin{aligned}
& D^{-1} \chi^{-2} \Phi\left(\chi^{2}, \xi^{2} ; u ; \tilde{\varphi}\right)=\sum_{n \geqslant 1} N_{n} b^{n}\left(\frac{\chi^{2}}{\lambda}\right)^{n(2 \mu+s-1)-\mu} \times \\
& \quad \times \int\left[\left\{Z_{\chi}\left(\eta_{1}(\varkappa)\right)\right\}^{2 n-1}-\left\{Z_{\chi}(\eta(\varkappa))\right\}^{2 n-1}\right]\left\{\frac{\bar{u}(\chi \varkappa)}{(2 \pi)^{d}} d \varkappa\right\}^{n},
\end{aligned}
$$

где

$$
\begin{gathered}
Z_{\chi}(z)=z^{\mu-1} \frac{\bar{K}_{1-\mu}(L \chi \sqrt{z})}{\bar{K}_{1-\mu}(L \sqrt{\lambda})}, \quad \bar{u}(k)=\frac{u(k)}{u(0)}, \\
\eta(\varkappa)=\varkappa^{2}-2 \varkappa \epsilon+1, \quad \epsilon=\frac{\mathbf{p}}{\chi}, \quad \eta_{1}(\varkappa)=\varkappa^{2}-2 \varkappa \epsilon_{1}, \quad \epsilon_{1}=\frac{\mathbf{p}_{1}}{\chi} .
\end{gathered}
$$

Поскольку параметр $\mu$ не зависит от переменных $p$ и $\xi$, то для определения его значения можно перейти к пределу $\xi \rightarrow 0$. В таком случае выражение $(84)$ преобразуется к виду

$$
\begin{aligned}
D^{-1} p^{-2} & \Phi\left(p^{2}, 0 ; u ; \tilde{\varphi}\right)=A_{1} b\left(\lambda l^{2}\right)^{1-\nu}\left(\frac{l}{L}\right)^{2 \nu} \bar{K}_{1-\mu}^{-1}(L \sqrt{\lambda})+ \\
& +A_{2} b^{2} \lambda^{1-\gamma} \bar{K}_{1-\mu}^{-3}(L \sqrt{\lambda})\left(p L^{\gamma}\right)^{-2}\left(\bar{K}_{\gamma}(0)-\bar{u}^{2}(p) \bar{K}_{\gamma}(p L)\right)+ \\
& +\sum_{n \geqslant 3} N_{n} b^{n}\left(\frac{p^{2}}{\lambda}\right)^{n(2 \mu+s-1)-\mu} \times \\
& \times \int\left[\left\{Z_{p}\left(\varkappa^{2}\right)\right\}^{2 n-1}-\left\{Z_{p}\left(\left(\varkappa-\epsilon_{0}\right)^{2}\right)\right\}^{2 n-1}\right]\left\{\frac{\bar{u}(p \varkappa)}{(2 \pi)^{d}} d \varkappa\right\}^{n},
\end{aligned}
$$

где

$$
\begin{gathered}
A_{1}=\frac{M \bar{K}_{\nu}(0)}{(2 \pi)^{s+1}}, \quad M=-\left.l^{-2} \frac{d \bar{u}(p)}{d p^{2}}\right|_{p=0}, \\
A_{2}=\frac{\Gamma^{3}(\nu)}{4(2 \pi)^{d} \Gamma(3 \nu)}, \quad \gamma=3 \mu+2 s-1, \quad\left|\epsilon_{0}\right|=1 .
\end{gathered}
$$


В выражении (87) указаны в явной форме лишь первые два члена ряда, представляющего функцию $D^{-1} p^{-2} \Phi\left(p^{2}, 0 ; u ; \tilde{\varphi}\right)$. Согласно $(77),(82),(83)$ и (85) исходное приближение $\widetilde{X}$ в рассматриваемом случае теперь имеет вид

$$
\widetilde{X}\left(\frac{p^{2}}{\lambda}, 0, b\right)=\left(\frac{p^{2}}{\lambda}\right)^{\mu} Z_{p}(1) .
$$

Далее, в уравнении (55) величину $X$ мы заменим выражением (90) и примем во внимание тот факт, что функция $\bar{K}_{1}(z)$ в малой окрестности точки $z=0$ ведет себя как

$$
\bar{K}_{1}(z)=1+\frac{z^{2}}{2} \ln z+O\left(z^{2}\right) .
$$

Поэтому в (87) положим $\gamma=1$ или

$$
\mu=\frac{2}{3}(1-s)
$$

что не противоречит условию (76). Тогда второй член в правой части равенства (87) примет вид

$$
A_{2} b^{2} \bar{K}_{1-\mu}^{-3}(L \sqrt{\lambda})(p L)^{-2}\left(1-\bar{u}^{2}(p) \bar{K}_{1}(p L)\right),
$$

где

$$
(p L)^{-2}\left(1-\bar{u}^{2}(p) \bar{K}_{1}(p L)\right) \sim-\frac{1}{2} \ln (p L)+O(1), \quad p \rightarrow 0,
$$

а степенная функция $\left(p^{2} / \lambda\right)^{n(2 \mu+s-1)-\mu}$ под знаком суммы по $n, n \geqslant 3$, будет равна $\left(p^{2} / \lambda\right)^{\mu(n-2) / 2}$. Если еше учесть условие $0<\bar{u}(p \varkappa) \leqslant 1$, то отсюда можно заключить, что любая частичная сумма ряда по $n \geqslant 3$ в (87) стремится к нулю при $p \rightarrow 0$. Кроме того, поскольку по условию нормировки в точке $p^{2}=\lambda$ должно выполняться равенство $\Phi(\lambda, 0 ; u ; \tilde{\varphi})=0$, то отсюда следует, что в рассматриваемом приближении правая часть уравнения (55) ведет себя в окрестности точки $p=0$ как

$$
1-\frac{1}{4} A_{2} b^{2} \bar{K}_{1-\mu}^{-3}(L \sqrt{\lambda}) \ln \frac{p^{2}}{\lambda}+O\left(p^{2} l^{2} \ln \frac{p^{2}}{\lambda}\right), \quad p \rightarrow 0 .
$$

Тогда, следуя изложенной выше схеме РГ-метода, коэффициент при $\ln \left(p^{2} / \lambda\right)$ в выражении (94) необходимо приравнять $\mu$. В результате мы находим связь между точкой нормировки $p^{2}=\lambda$ и параметром $a$,

$$
a=\alpha_{s}\left(\lambda l^{2}\right)^{1-s},
$$

где

$$
\alpha_{s}=(4 \pi)^{s+1}\left(\frac{\mu \Gamma^{3}(1-\mu) \Gamma(3 \nu)}{\Gamma^{3}(\nu)}\right)^{1 / 2}, \quad \nu=\frac{s+2}{3} .
$$

Отметим при этом, что $\alpha_{s} \rightarrow 0$, когда $s \rightarrow 1(d \rightarrow 4)$. 
Для определения асимптотики функции $g(r, t)$, когда $r \gg L, t \rightarrow \infty$, обратимся к формуле (46) и запишем ее в виде

$$
\tilde{g}(t, r)=e^{E_{0} t} \frac{1}{2 \pi i} \int_{E_{0}-i \infty}^{E_{0}+i \infty} e^{\left(E-E_{0}\right) t} \tilde{\psi}(r, \xi) d E
$$

где функция $\tilde{\psi}(r, \xi)$ определена в $(80)$, а связь между переменными $E$ и $\xi$ осуществляется посредством тождества (50), которое в принятом здесь приближении имеет вид

$$
\begin{aligned}
E-E_{0}= & D \xi^{2}+\left[S_{1}\left(p_{1}, \xi\right)-S_{1}(0,0)\right]+\left[S_{2}\left(p_{1}, \xi\right)-S_{2}(0,0)\right]+ \\
& +D \xi^{2} \sum_{n \geqslant 3} N_{n} b^{n}\left(\frac{\xi^{2}}{\lambda}\right)^{\mu(n-2) / 2} \times \\
& \times \int\left[\left\{Z_{\xi}\left(\eta_{0}(\varkappa)\right)\right\}^{2 n-1}-\left\{Z_{\xi}\left(\varkappa^{2}\right)\right\}^{2 n-1}\right]\left\{\frac{\bar{u}(\xi \varkappa)}{(2 \pi)^{d}} d \varkappa\right\}^{n},
\end{aligned}
$$

где

$$
\begin{gathered}
{\left[S_{1}\left(p_{1}, \xi\right)-S_{1}(0,0)\right]=-k_{s}\left(D w l^{d}\right)^{1 / 3} \xi^{2 \nu}+O\left(M l^{2} D \xi^{2}\right),} \\
k_{s}=\frac{4^{1-s} \Gamma(1+\mu / 2)}{\Gamma(2-\mu / 2) \Gamma^{2}(1-\mu)}\left(\frac{\Gamma(2+s)}{2 \pi^{1+s} \mu^{2}}\right)^{1 / 3}, \\
{\left[S_{2}\left(p_{1}, \xi\right)-S_{2}(0,0)\right]=O\left((1-s) D \xi^{2}\right) .}
\end{gathered}
$$

В итоге мы приходим к следуюшей связи между переменными $E-E_{0}$ и $\xi$ :

$$
E_{0}-E=k_{s}\left(D w l^{d}\right)^{1 / 3} \xi^{2 \nu}+O\left(D \xi^{2} \max \left(C, M l^{2}\right)\right), \quad \xi \rightarrow 0
$$

Теперь для асимптотической оценки интеграла в формуле (96), когда значения переменных $r$ и $t$ стремятся к бесконечности, но их отношение $r / t$ фиксировано и $r / t \ll 1$, мы воспользуемся методом перевала. В результате получим для $\tilde{g}(r, t)$ асимптотическое выражение

$$
\tilde{g}(r, t) \sim \pm \sum_{n} \frac{C_{d}}{w l^{d} t^{2}} \Lambda^{\mu / 2}(r, t) \exp \left[E_{0} t-\eta_{n} \Lambda(r, t)+i \delta_{n}\right]
$$

в котором

$$
\Lambda(r, t)=\left[\frac{r^{d+2}}{D w l^{d} t^{3}}\right]^{1 /(d-1)}, \quad \eta_{n}=c_{d} \exp \left(3 \pi i \frac{2 n+1}{d-1}\right), \quad \delta_{n}=\frac{\pi}{2} \frac{d+2+6 n}{d-1},
$$

$C_{d}$ и $c_{d}$ суть положительные постоянные, пропорциональные $(4-d)^{d /(d-1)}$ и $(4-d)^{2 /(d-1)}$, соответственно, а знак перед суммой в (99) выбирается так, чтобы правая часть (99) принимала неотрицательные значения. В случаях $d=2(s=0)$ и $d=3$ $(s=1 / 2)$ отсюда находим

$$
\tilde{g}(r, t) \sim \begin{cases}\frac{C_{2}}{w l^{2} t^{2}}\left(\frac{r^{4}}{D w l^{2} t^{3}}\right)^{1 / 3} \exp \left(E_{0} t+\frac{c_{2} r^{4}}{D w l^{2} t^{3}}\right), & \text { если } d=2, \\ \frac{C_{3}}{w l^{3} t^{2}}\left(\frac{r^{5}}{D w l^{3} t^{3}}\right)^{1 / 12} \exp \left(E_{0} t\right)\left|\cos \left[c_{3}\left(\frac{r^{5}}{D w l^{3} t^{3}}\right)^{1 / 2}+\frac{\pi}{4}\right]\right|, & \text { если } d=3 .\end{cases}
$$


Из формул (99) и (100) непосредственно следует, что, несмотря на малость параметра $a$, характеризуюшую формально преобладание механизма диффузии в эволюции системы, влияние корреляционного эффекта на асимптотику функции $\tilde{g}(r, t)$ в случае $d<4$ оказывается очень сушественным и, по-видимому, им можно пренебречь лишш при $d>4$. Уместно отметить, что в противоположном случае $a \gg 1$ искомая асимптотика определяется главным образом корреляционным эффектом, следствием чего в показателе ее экспоненты будет фигурировать величина, пропорциональная $t^{2}$.

В заключение укажем на важное следствие, вытекающее из формулы (99). Согласно определению функции $\Lambda(r, t)$ ее область определения можно разделить линией $r_{d}(t)=$ $\left(D w l^{d} t^{3}\right)^{1 /(d+2)}$ на две части, в одной из которых (где $\left.r<r_{d}(t)\right)$ реализуется асимптотический режим $\Lambda(r, t) \rightarrow 0$, а в другой (где $\left.r>r_{d}(t)\right)-\Lambda(r, t) \rightarrow \infty$. Эти два предельных случая приводят соответственно к различным асимптотическим выражениям функции $\tilde{g}(r, t)$. При этом показатель степени $3 /(d+2)$ в функциональной зависимости $r_{d}(t)$ известен в некоторых задачах статистической физики как один из их критических индексов, что, по-видимому, свидетельствует о родстве с ними рассматриваемой здесь задачи.

Благодарности. Автор искренне благодарен Л. В. Богачеву, Р. А. Минлосу, Е. А. Печерскому, С. А. Пирогову, Д. Д. Соколову и Е. Б. Яровой за полезные обсуждения изложенных здесь результатов.

\section{Список литературы}

[1] Ya. B. Zeldovich, S. A. Molchanov, A. A. Ruzmaikin, D. D. Sokoloff. Sov. Sci. Rev. C. 1988. V. 7. P. $1-110$.

[2] S. A. Molchanov. Lectures on the random media. In: Lectures on Probability Theory. Lect. Notes Math. V. 1581. Eds. D. Bakry et al. Berlin: Springer, 1994. P. 242-411.

[3] Л. Б. Богачев, С. А. Молчанов. ТМФ. 1989. Т. 81. С. 281-290; 1990. Т. 82. С. 143-154; 1991. T. 87. C. $254-273$.

[4] S. Albeverio, L. V. Bogachev, S. A. Molchanov, E. B. Yarovaya. Markov Processes and Related Fields. 2000. V. 6. P. 473-516.

[5] Р. Фейнман. Статистическая механика. М.: Мир, 1978.

[6] Я. Б. Зельдович, С. А. Молчанов, А. А. Рузмайкин, Д. Д. Соколов. УФН. 1987. Т. 152. C. $3-32$.

[7] М. Кац. Вероятность и смежные вопросы в физике. М.: Мир, 1965.

[8] Дж. Глимм, А. Джаффе. Математические методы квантовой физики. (Подход с использованием функциональных интегралов). М.: Мир, 1984.

[9] P. Cartier, C. DeWitt-Morette. A Rigorous Mathematical Foundation of Functional Integration. New York: Plenum Press, 1997.

[10] L. S. Schulman. Techniques and Applications of Path Integration. New York: Wiley, 1981.

[11] В. И. Алхимов. ТМФ. 1976. Т. 29. С. 424-429.

[12] В. И. Алхимов. ТМФ. 1979. Т. 39. С. 215-218.

[13] D. C. Brydges. A short course on cluster expansions. In: Critical Phenomena, Random Systems, Gauge Theories. Les Houches Summer School (1984). Eds. K. Osterwalder, R. Stora. Amsterdam: North Holland, 1986. P. 131-183.

[14] Э. З. Кучинский, М. В. Садовский. ЖЭТФ. 1998. Т. 113. С. 664-678.

[15] Н. Н. Боголюбов, Д. В. Ширков. Введение в теорию квантованных полей. М.: Наука, 1984.

[16] М. Абрамовиц, И. Стиган. Справочник по специальным функциям. М.: Наука, 1979. 